CONVEX AND FRACTAL GEOMETRY

BANACH CENTER PUBLICATIONS, VOLUME 84

INSTITUTE OF MATHEMATICS

POLISH ACADEMY OF SCIENCES

WARSZAWA 2009

\title{
MINIMAL PAIRS OF BOUNDED CLOSED CONVEX SETS AS MINIMAL REPRESENTATIONS OF ELEMENTS OF THE MINKOWSKI-RÅDSTRÖM-HÖRMANDER SPACES
}

\author{
JERZY GRZYBOWSKI \\ Faculty of Mathematics and Computer Science, Adam Mickiewicz University \\ Umultowska 87, 61-614 Poznań, Poland \\ E-mail: jgrz@amu.edu.pl \\ DIETHARD PALLASCHKE \\ Institut für Statistik und Mathematische Wirtschaftstheorie, Universität Karlsruhe \\ Kaiserstr. 12, D-76128 Karlsruhe, Germany \\ E-mail:lh09@rz.uni-karlsruhe.de \\ RYSZARD URBAŃSKI \\ Faculty of Mathematics and Computer Science, Adam Mickiewicz University \\ Umultowska 87, 61-614 Poznań, Poland \\ E-mail: rich@amu.edu.pl
}

\begin{abstract}
The theory of minimal pairs of bounded closed convex sets was treated extensively in the book authored by D. Pallaschke and R. Urbański, Pairs of Compact Convex Sets, Fractional Arithmetic with Convex Sets ([27]). In the present paper we summarize the known results, generalize some of them and add new ones.
\end{abstract}

\section{Introduction}

1.1. Minkowski-Rådström-Hörmander space. Let $X=(X, \tau)$ be a real Hausdorff topological vector space and let $\mathcal{B}(X)$ be the family of all nonempty bounded closed convex subsets of $X$. For $A, B \in \mathcal{B}(X), \alpha \in \mathbb{R}_{+}$the Minkowski sum $A+B=\{a+b \mid a \in A, b \in B\}$, the closure of the Minkowski sum $A+B=\overline{A+B}$ and $\alpha \cdot A=\{\alpha a \mid a \in A\}$. The addition "+" and the multiplication "." turn the family $\mathcal{B}(X)$ into a cone.

If $A+B=C$ then the sets $A$ and $B$ are summands of the set $C$. The set $C$ is an anti-summand of $A$ and of $B$.

2000 Mathematics Subject Classification: Primary 26A27; Secondary 58C27, 90C30.

Key words and phrases: minimal pairs of convex sets, reduced pairs.

The paper is in final form and no version of it will be published elsewhere. 
By $A-B=\{x \in X \mid x+B \subset A\}$, we denote the Minkowski difference of $A$ and $B$, [31]. The set $A \dot{-} B$ can be empty. If $B$ is a summand of $A$ then $(A-B)+B=A$.

In the cone $\mathcal{B}^{2}(X)$ the relation

$$
(A, B) \sim(C, D) \Leftrightarrow A \dot{+} D=B \dot{+} C
$$

is an equivalence relation and $[A, B]$ denotes the quotient class of the pair $(A, B)$ in $\mathcal{B}^{2}(X)$. The quotient set $\widetilde{X}=\mathcal{B}^{2}(X) / \sim$ with the addition $[A, B]+[C, D]=[A \dot{+} C, B \dot{+} D]$ and the multiplication $\alpha \cdot[A, B]=\left[\alpha^{+} A \dot{+} \alpha^{-} B, \alpha^{+} B \dot{+} \alpha^{-} A\right]$, where $\alpha^{+}=\max (0, \alpha)$, $\alpha^{-}=\max (0,-\alpha)$, is a vector space called the Minkowski-Rådström-Hörmander space over $X$.

The zero vector is $\tilde{0}=[\{0\},\{0\}]$ and the opposite vector is $-[A, B]=[B, A]$. The mapping $\mathcal{B}(X) \ni A \mapsto[A,\{0\}] \in \widetilde{X}$ is the natural embedding of the cone $\mathcal{B}(X)$ into $\widetilde{X}$.

For two bounded closed convex sets $A, B \subset X$ we will use the notation

$$
A \vee B=\overline{\operatorname{conv}}(A \cup B) \text {. }
$$

In [25] the following notion was introduced: Let $A, B, S \in \mathcal{B}(X)$; then we say that $S$ separates the sets $A$ and $B$ if for every $a \in A$ and $b \in B$ we have $(a \vee b) \cap S \neq \emptyset$. The following theorem is important and very useful:

TheOREm 1.1 ([35]). Let $X$ be a real topological vector space and $A, B \in \mathcal{B}(X)$. Then the following statements are equivalent:

i) The set $A \cup B$ is convex.

ii) The set $A \cap B$ separates the sets $A$ and $B$.

iii) The set $A \vee B$ is a summand of the set $A \dot{+} B$.

iv) $A \dot{+} B=A \vee B \dot{+} A \cap B$ and $A \cap B \neq \emptyset$.

Let us state explicitly the order cancellation law (see [20], [29], [34]).

Let $X$ be a real topological vector space and $A, B, C$ be subsets of $X$ such that $A$ is nonempty and bounded and $C$ is closed and convex. Then the inclusion $A+B \subset A+C$ holds if and only if $B \subset C$.

A. G. Pinsker, [28], introduced the following partial order in $\widetilde{X}$ :

$$
[A, B] \preceq[C, D] \Leftrightarrow A \dot{+} D \subset B \dot{+} C .
$$

This partial ordering is independent of a specific choice of representatives.

Moreover A. G. Pinsker showed also that with respect to the partial order $\preceq$ in $\widetilde{X}$, the supremum "sup" exists and that

$$
\sup \{[A, B],[C, D]\}=[(A \dot{+} D) \vee(C \dot{+} B), B \dot{+} D] \in \widetilde{X} .
$$

The operation "sup" is also independent of the choice of representatives.

The ordered space $(\widetilde{X}, \preceq)$ is called the Minkowski-Rådström-Hörmander lattice.

Let us note that the Minkowski-Rådström-Hörmander lattice has many interesting properties. In particular, it is a basic tool in the quasidifferential calculus of V. F. Demyanov and A. M. Rubinov (see [5], [6] and [7]). The basic facts of this theory and its role in nonsmooth analysis can be found in Chapter 10 of the book by D. Pallaschke and R. Urbański, [27]. 
1.2. Minkowski duality. Let $(Y,\|\cdot\|)$ be a Banach space and $Y^{*}$ be the dual space, i.e. the linear space of all continuous linear functionals defined on $Y$.

By $\mathcal{S}(Y)$ we denote the family of all continuous sublinear (that is subadditive and positively homogeneous) functions $p: Y \rightarrow \mathbb{R}$. For $p \in \mathcal{S}(Y)$, the set $\left.\partial p\right|_{0}=\left\{f \in Y^{*} \mid f \leq p\right\}$ $\in \mathcal{B}\left(Y^{*}\right)$ is called the subdifferential of $p$ in $0 \in Y$.

In fact $\left.\partial p\right|_{0}$ is closed and therefore compact in the weak-*-topology. By $\mathcal{K}\left(Y^{*}\right) \subset$ $\mathcal{B}\left(Y^{*}\right)$, we denote the family of all nonempty weak-*-compact convex subsets of $Y^{*}$.

On the other hand, for any $A \in \mathcal{K}\left(Y^{*}\right)$, the function

$$
p_{A}: Y \rightarrow \mathbb{R} \text { given by } p_{A}(x)=\sup _{f \in A} f(x)
$$

is continuous and sublinear and it is called the support function of the set $A$.

The operations of taking the subdifferential of sublinear function and of taking the support function of bounded closed convex set are mutual inverses and establish Minkowski duality between the cones $\mathcal{K}\left(Y^{*}\right)$ and $\mathcal{S}(Y)$.

It was shown by L. Hörmander [20] that for a locally convex topological space $X$ the support function $p_{A}$ of a bounded closed convex set $A \in \mathcal{B}(X)$ defined by $p_{A}(f)=$ $\sup _{x \in A} f(x)$ is sublinear and continuous with respect to the weak-*-topology on $X^{*}$.

Let us denote by

$$
\mathcal{D S}(Y)=\{\varphi=p-q \mid p, q \in \mathcal{S}(Y)\}
$$

the real vector space of differences of continuous sublinear functions.

The Minkowski duality naturally extends to the isomorphic mapping between the lattice $\mathcal{D} \mathcal{S}(Y)$ and the Minkowski-Rådström-Hörmander lattice $\mathcal{K}^{2}\left(Y^{*}\right) / \sim$.

1.3. Minimal pairs. We assume that $(X, \tau)$ is a real Hausdorff topological vector space. We introduce the partial order in $\mathcal{B}^{2}(X)$ by $(A, B) \leq(C, D)$ if and only if $(A, B) \sim(C, D)$ and $A \subset C, B \subset D$. (Thanks to the order cancellation law $(A, B) \sim(C, D)$ and $A \subset C$ imply that $B \subset D$.)

The pair $(A, B) \in \mathcal{B}^{2}(X)$ is called a minimal pair if it is minimal with respect to the ordering " $\leq "$. So we look for minimal representatives of every quotient class $[A, B]$.

Let us fix some notations. If $f \in X^{*}$ is a continuous linear functional and $A \in \mathcal{B}(X)$ then we denote by

$$
H_{f}(A)=\left\{a \in A \mid f(a)=\sup _{b \in A} f(b)\right\}
$$

the face of $A$ with respect to $f$.

The face $H_{f}(A)$ is a facet of $A$ if the smallest affine subspace containing $H_{f}(A)$ has codimension one in the smallest affine subspace containing $A$.

For the sum of the faces of two nonempty compact convex sets $A, B \subset X$ with respect to $f \in X^{*}$, the following identity holds:

$$
H_{f}(A+B)=H_{f}(A)+H_{f}(B) .
$$

Without the assumption of compactness the faces can be empty sets.

For $A \in \mathcal{B}(X)$, we denote by ext $A$ the set of extreme points of $A$ and by $\exp A$ the set of its exposed points. Recall that $x_{0} \in A$ is an exposed point if and only if there exists an $f \in X^{*} \backslash\{0\}$ such that $H_{f}(A)=\left\{x_{0}\right\}$. 
2. Existence of minimal pairs. The following theorem on the existence of minimal pairs was proved by D. Pallaschke, S. Scholtes and R. Urbański in [24]:

THEOREM 2.1. Let $X$ be a real Hausdorff topological vector space and $A, B \in \mathcal{B}(X)$. If the sets $A, B$ are compact then there exists a minimal pair $(C, D) \in[A, B]$ such that $(C, D) \leq(A, B)$.

In the proof of this theorem, the Kuratowski-Zorn Lemma is applied. In [13], Theorem 2.1. was used to prove the following:

TheOrem 2.2. Let $X$ be a reflexive Banach space and $A, B \in \mathcal{B}(X)$. Then there exists a minimal pair $(C, D) \in[A, B]$ such that $(C, D) \leq(A, B)$.

Theorem 2.2 motivates the following:

Problem 2.3. Let $Y^{*}$ be the dual space of a Banach space $Y$ and $A, B \in \mathcal{B}\left(Y^{*}\right)$ be weak*-closed sets. Does there exist a minimal pair $(C, D) \in[A, B]$ such that $(C, D) \leq(A, B)$ ?

Theorem 2.2 is not true for some Banach spaces. The following example was given in [13].

EXAMPLE 2.4. Let $c_{0}$ be the space of all real sequences tending to 0 with the supremum norm. Let $A=\left\{\left(a_{i}\right)_{i \in \mathbb{N}} \mid 0 \leq a_{i} \leq 1\right.$ for all $\left.i \in \mathbb{N}\right\}$ and $B=-A$. The sets $A$ and $B$ belong to $\mathcal{B}\left(c_{0}\right)$. In [13], it is proved that the quotient class $[A, B]$ in $\widetilde{c_{0}}$ does not have a minimal element. Also, the quotient class $[A, B]$ in $\widetilde{l^{\infty}}$ does not have a minimal element.

Problem 2.5. Is the existence of minimal pairs in each quotient class $\tilde{x}$ in the Minkowski-Rådström-Hörmander space $\widetilde{X}$ over a Banach space $X$ equivalent to the reflexivity of $X$ ? Is there a quotient class $[A, B]$ in $\widetilde{l^{1}}$ that has no minimal pair?

3. Uniqueness of minimal pairs in $\mathbb{R}^{2}$. The uniqueness of a minimal pair up to translation in every quotient class in $\widetilde{\mathbb{R}}$ is quite obvious since only pairs where one set is a singleton are minimal pairs. S. Scholtes, [32] and J. Grzybowski, [10] proved the following independently:

Theorem 3.1. Let $(A, B),(C, D) \in \mathcal{B}^{2}\left(\mathbb{R}^{2}\right)$ be two minimal pairs. If $[A, B]=[C, D]$, then there exists a vector $x \in \mathbb{R}^{2}$ such that $C=A+x$ and $D=B+x$.

Let us consider the following two properties of the pair $(A, B) \in \mathcal{B}^{2}(X)$ :

$(\mathcal{P} 1)$ Let $C, D \in \mathcal{B}(X)$ be two minimal (with respect to the inclusion) anti-summands of both $A$ and $B$. Then there exists a vector $x \in X$ such that $D=C+x$.

$(\mathcal{P} 2)$ Let $C, D \in \mathcal{B}(X)$ be two maximal (with respect to the inclusion) summands of both $A$ and $B$. Then there exists a vector $x \in X$ such that $D=C+x$.

In [18], it was shown that the mapping $[A, B] \ni(C, D) \mapsto A \dot{+} D \in \mathbf{a}(A, B)$ is an isomorphism between two partially ordered sets, the quotient class $([A, B], \leq)$ and the family $\mathbf{a}(A, B)$ of all common anti-summands of $A$ and $B$. This property, $(\mathcal{P} 1)$, of the pair $(A, B)$ is equivalent to the uniqueness up to translation of minimal pairs in $[A, B]$. If minimal pairs in $[A, B]$ are unique up to translation we say that the class $[A, B]$ has the property of translation. 
S. Scholtes proved (Proposition 2.2 in [33]) that for a given space $X$, if all pairs of compact sets $(A, B) \in \mathcal{B}^{2}(X)$ have property $(\mathcal{P} 2)$ then they also have property $(\mathcal{P} 1)$. Therefore, the following theorem proved by J. Grzybowski in [11] is stronger than Theorem 3.1.

TheOREM 3.2. Let $(A, B) \in \mathcal{B}^{2}\left(\mathbb{R}^{2}\right)$. Then the pair $(A, B)$ has property $(\mathcal{P} 2)$.

It is convenient to present here the general approach applied first in [10] and later developed in [11].

Let $\mathcal{A}$ be the cone of normalized nondecreasing real functions on the interval $[0,2 \pi]$, that is, for $f \in \mathcal{A}$ we have $f(0)=0$ and $f(t)=f\left(t^{+}\right)=\lim _{s \rightarrow t^{+}} f(s)$, for $t \in[0,2 \pi)$. In other words, $\mathcal{A}$ is the cone of positive elements in the lattice $N B V[0,2 \pi]$ of normalized functions of bounded variations. For the functions $f, g \in \mathcal{A}$, by $\min (f, g)$ we denote the greatest function in $\mathcal{A}$ such that $f-\min (f, g), g-\min (f, g) \in \mathcal{A}$.

For convenience we denote $e^{i t}=(\cos t, \sin t) \in \mathbb{R}^{2}$. For $A \in \mathcal{B}\left(\mathbb{R}^{2}\right), u \in \mathbb{R}^{2}$ we have the face $H_{\langle u, \cdot\rangle}(A)=\left\{a \in A \mid\langle u, a\rangle=\max _{b \in A}\langle u, b\rangle\right\}$, and the boundary function $h_{A}$ : $[0,2 \pi] \rightarrow \partial A$ defined by $h_{A}=H_{\left\langle e^{i\left(t+\frac{\pi}{2}\right)}, \cdot\right\rangle}\left(H_{\left\langle e^{i t}, \cdot\right\rangle}(A)\right)$. We also need the arc length function $f_{A}:[0,2 \pi] \rightarrow \mathbb{R}_{+}$where $f_{A}(t)$ is the length of the arc contained in $\partial A$ joining $h_{A}(0)$ and $h_{A}(t)$. The function $f_{A}$ belongs to $\mathcal{A}$. The mapping $\mathcal{B}\left(\mathbb{R}^{2}\right) \ni A \mapsto f_{A} \in \mathcal{A}$ is a homomorphism of real cones.

For a nondecreasing function $f \in \mathcal{A}$ we define the function $h_{f}:[0,2 \pi] \rightarrow \mathbb{R}^{2}$ by $h_{f}(t)=\int_{0}^{t} e^{i\left(s+\frac{\pi}{2}\right)} d f(s)$, where the latter is the Stieltjes integral.

If $h_{f}(2 \pi)=0$ then we denote $A_{f}=\overline{\operatorname{conv}} h_{f}([0,2 \pi])$. Then $A_{f_{A}}=A-h_{A}(0)$ and $f_{A_{f}}=f$. If $h_{f}(2 \pi) \neq 0$ then there exists the unique function $g \in \mathcal{A}$ which takes exactly two values such that $h_{g}(2 \pi)=-h_{f}(2 \pi)$. Hence $h_{f+g}(2 \pi)=0$. Then there exists $A_{f+g}$.

The following theorem describes the construction of a minimal pair equivalent to the given one (see [10]):

TheOREM 3.3. Let $(A, B) \in \mathcal{B}^{2}\left(\mathbb{R}^{2}\right)$. Let $g$ be a function in $\mathcal{A}$ such that $g$ takes not more than two values and $h_{g}(2 \pi)=h_{\min \left(f_{A}, f_{B}\right)}(2 \pi)$. Denote $f_{1}=f_{A}-\min \left(f_{A}, f_{B}\right)+g$ and $f_{2}=f_{B}-\min \left(f_{A}, f_{B}\right)+g$. Then the pair $\left(A_{f_{1}}+h_{A}(0), A_{f_{2}}+h_{B}(0)\right)$ is minimal and belongs to $[A, B]$.

Theorem 3.4, the Criterion of Minimality (see Lemma 5.1 in [10]) follows from Theorem 3.3 .

TheOrem 3.4. Let $(A, B) \in \mathcal{B}^{2}\left(\mathbb{R}^{2}\right)$. The pair $(A, B)$ is minimal if and only if the function $\min \left(f_{A}, f_{B}\right)$ takes at most two values.

The following is a corollary of the previous theorem, the Criterion of Minimality.

THEOREM 3.5. Let $(A, B)$ be a pair of convex polygons in $\mathbb{R}^{2}$. The pair $(A, B)$ is minimal if and only if $A$ and $B$ have at most one pair of parallel edges that lie on the same side of the polygons.

4. Reduced pairs. The notion of reduced pairs was introduced by Ch. Bauer in [3].

Definition. The pair $(A, B) \in \mathcal{B}^{2}(X)$ is called reduced if $A+B$ is a summand of $C$ whenever $A$ and $B$ are summands of $C$. 
The pair $(A, B)$ is reduced if and only if $[A, B]=\{(A \dot{+} C, B \dot{+} C) \mid C \in \mathcal{B}(X)\}$. All reduced pairs of sets are minimal, but not all minimal pairs are reduced. However, all minimal pairs in $\mathcal{B}^{2}(\mathbb{R})$ are reduced. In $\mathbb{R}^{2}$ the following theorem (see also Theorem 4.2 in [3]) holds true:

THEOREM 4.1. Let $(A, B) \in \mathcal{B}^{2}\left(\mathbb{R}^{2}\right)$. Then the pair $(A, B)$ is reduced if and only if $\min \left(f_{A}, f_{B}\right) \equiv 0$.

Proof. Let $(A, B) \in \mathcal{B}^{2}\left(\mathbb{R}^{2}\right)$ and $\min \left(f_{A}, f_{B}\right) \equiv 0$. Let $(C, D) \in[A, B]$. Then $f_{A}+$ $f_{D}=f_{B}+f_{C}$. By Remark 2.4 and Theorem 2.5 in [10], $f_{A}=f_{A}-f_{\min \left(f_{A}, f_{B}\right)}=$ $f_{C}-f_{\min \left(f_{C}, f_{D}\right)}$ and $f_{B}=f_{B}-f_{\min \left(f_{A}, f_{B}\right)}=f_{D}-f_{\min \left(f_{C}, f_{D}\right)}$. Since $f_{A}+f_{\min \left(f_{C}, f_{D}\right)}=$ $f_{C}, h_{\min \left(f_{C}, f_{D}\right)}(2 \pi)=0$. Hence, $C=A_{f_{C}}+h_{C}(0)=A_{f_{A}}+A_{\min \left(f_{C}, f_{D}\right)}+h_{C}(0)=$ $A+A_{\min \left(f_{C}, f_{D}\right)}+h_{C}(0)-h_{A}(0)$. Also, $D=A+A_{\min \left(f_{C}, f_{D}\right)}+h_{D}(0)-h_{B}(0)$. The fact that $h_{A}(0)+h_{D}(0)=h_{B}(0)+h_{C}(0)$ completes the proof that the pair $(A, B)$ is reduced.

On the other hand, let $\min \left(f_{A}, f_{B}\right) \neq 0$. If the pair $(A, B)$ is not minimal then it is not reduced. Assume that the pair $(A, B)$ is minimal. By Theorem 3.4 the function $\min \left(f_{A}, f_{B}\right)$ takes two values. It implies that the boundaries of $A$ and $B$ contain coparallel edges (parallel and lying on the same side of sets). In fact, for some $a>0$ and $\alpha \in(0,2 \pi]$

$$
\min \left(f_{A}, f_{B}\right)(t)= \begin{cases}0 & \text { if } t<\alpha \\ a & \text { if } t \geq \alpha .\end{cases}
$$

Let $g$ be the function in $\mathcal{A}$ defined by

$$
g(t)=\left\{\begin{array}{lll}
0 & \text { if } \quad t<\alpha-\frac{\pi}{3} \\
a & \text { if } \quad \alpha-\frac{\pi}{3} \leq t<\alpha+\frac{\pi}{3} \\
2 a & \text { if } \quad t \geq \alpha+\frac{\pi}{3} .
\end{array}\right.
$$

If $\alpha \leq \frac{\pi}{3}$, then instead $\alpha-\frac{\pi}{3}$ we take $\alpha-\frac{\pi}{3}+2 \pi$; if $\alpha>2 \pi-\frac{\pi}{3}$, then instead $\alpha+\frac{\pi}{3}$ we take $\alpha+\frac{\pi}{3}-2 \pi$. We have $h_{\min \left(f_{A}, f_{B}\right)}(2 \pi)=h_{g}(2 \pi)$. Denote $C=A_{f_{A}+g-\min \left(f_{A}, f_{B}\right)}+h_{A}(0)$ and $D=A_{f_{B}+g-\min \left(f_{A}, f_{B}\right)}+h_{B}(0)$. Hence $(C, D) \in[A, B]$, however, since $f_{C}-f_{A}$ is not a nondecreasing function, the set $A$ is not a summand of $C$ and the pair $(A, B)$ is not reduced.

A simple example of a minimal pair that is not reduced is a pair consisting of any triangle and any one of its sides.

Theorem 4.1 implies the following corollary analogous to Theorem 3.5.

Corollary 4.2. Let $(A, B)$ be a pair of convex polygons in $\mathbb{R}^{2}$. The pair $(A, B)$ is reduced if and only if they have no pair of parallel edges that lie on the same side of the polygons.

Let $(A, B)$ be two polytopes in $\mathbb{R}^{n}$. We call an edge (one-dimensional face) $k$ of $A$ and an edge $l$ of $B$ equiparallel if $k=H_{f}(A)$ and $l=H_{f}(B)$ for some linear functional $f \in\left(\mathbb{R}^{n}\right)^{*}$.

Ch. Bauer in [3] characterized all reduced pairs of polytopes in the following theorem:

TheOREM 4.3. Let $(A, B)$ be a pair of polytopes in $\mathbb{R}^{n}$. The pair $(A, B)$ is reduced if and only if $A$ and $B$ have no equiparallel edges.

The following propositions were given in [17] as Propositions 2 and 3: 
Proposition 4.4. Let $X$ be a locally convex space and $A$ be a segment in $X$ and $B \in$ $\mathcal{B}(X)$. The pair $(A, B)$ is minimal if and only if for any $\varepsilon>0$ there exists $f \in X^{*}, f \neq 0$ such that $H_{f}(A)=A$ and $H_{f}(B)$ does not contain any translate of $\varepsilon A$.

Proposition 4.5. Let $A$ be a segment in $\mathbb{R}^{n}$ and $B \in \mathcal{B}\left(\mathbb{R}^{n}\right)$. If $(A, B)$ is minimal then $(A, B)$ has the property of translation.

Proof. Let $(C, D)$ be equivalent to $(A, B)$. Then for all $f \in\left(\mathbb{R}^{n}\right)^{*}, f \neq 0$, if $H_{f}(A)=A$ then $A+H_{f}(D)=H_{f}(B)+H_{f}(C)$. The family $F_{m}$ of all $f \in\left(\mathbb{R}^{n}\right)^{*}$ such that $H_{f}(C)$ does not contain any translate of the segment $\frac{1}{m} A$ is open in $\left(\mathbb{R}^{n}\right)^{*}$. Then there exists $f_{m}$ such that $H_{f_{m}}(B+C)$ is a segment, $A+H_{f_{m}}(D)=H_{f_{m}}(B)+H_{f_{m}}(C)$ and $H_{f_{m}}(B)$ does not contain any translate of $\frac{1}{m} A$. Hence $H_{f_{m}}(C)$ is a segment which contains some translate of $\left(1-\frac{1}{m}\right) A$. Since $C$ is compact, $C$ contains some translate of $A$.

The following new theorem is stronger than Theorem 2 in [17] which proves only the minimality of a pair of sets. Theorem 4.6 generalizes the sufficient condition of reducibility given in Theorem 4.3.

TheOREM 4.6. Let $A, B \in \mathcal{B}\left(\mathbb{R}^{n}\right)$ and $A$ be a polytope such that for all $f \in\left(\mathbb{R}^{n}\right)^{*}$, if $H_{f}(A)$ is a segment, then $\left(H_{f}(A), H_{f}(B)\right)$ is a minimal pair. Then the pair is $(A, B)$ reduced.

Proof. Let $(C, D) \in[A, B]$. For all $f \in\left(\mathbb{R}^{n}\right)^{*}$, if $H_{f}(A)$ is a segment, then $\left(H_{f}(C), H_{f}(D)\right)$ $\sim\left(H_{f}(A), H_{f}(B)\right)$ and, by Proposition 4.5, $H_{f}(C)$ contains a translate of $H_{f}(A)$. By Theorem 3.2.8 in [31], $A$ is a summand of $C$; let us say $C=A+M$. Then $D=$ $B+M$.

The following corollary from Theorem 4.6 on reduced pairs was proved in [15]:

Corollary 4.7. Let $(A, B) \in \mathcal{B}^{2}\left(\mathbb{R}^{n}\right)$ and $A$ be a polytope. If, for all $f \in\left(\mathbb{R}^{n}\right)^{*}$ such that $H_{f}(A)$ is an edge of $A$, the face $H_{f}(B)$ is a singleton. Then the pair $(A, B)$ is reduced.

The following proposition can be easily proved:

Proposition 4.8. Let $X$ be a locally convex space and $A$ be a polytope in $X$ and $B \in$ $\mathcal{B}(X)$. For all $f \in X^{*}$, if $H_{f}(A)$ is a segment then $H_{f}(B)$ is a singleton. Then $(A, B)$ is a minimal pair.

EXAmPLE 4.9. Let us consider the following polytopes $A, B \in \mathcal{B}\left(\mathbb{R}^{3}\right)$. Let $A$ be the truncated octahedron with the vertices $a_{1}, \ldots, a_{24}$ and $B$ be its subset with the vertices $b_{1}, \ldots, b_{36}$ (see Figure 1 ).

$$
\begin{array}{llll}
a_{1}=(-6,12,0), & a_{2}=(0,12,6), & a_{3}=(6,12,0), & a_{4}=(-12,6,0), \\
a_{5}=(0,6,12), & a_{6}=(12,6,0), & a_{7}=(6,0,12), & a_{8}=(12,0,6), \\
b_{1}=(-3,11,-3), & b_{2}=(3,11,3), & b_{3}=(-4,10,4), & b_{4}=(-8,8,-2), \\
b_{5}=(2,8,8), & b_{6}=(8,8,2), & b_{7}=(-10,4,4), & b_{8}=(-4,4,10), \\
b_{9}=(-11,3,-3), & b_{10}=(3,3,11), & b_{11}=(11,3,3), & b_{12}=(8,2,8) .
\end{array}
$$




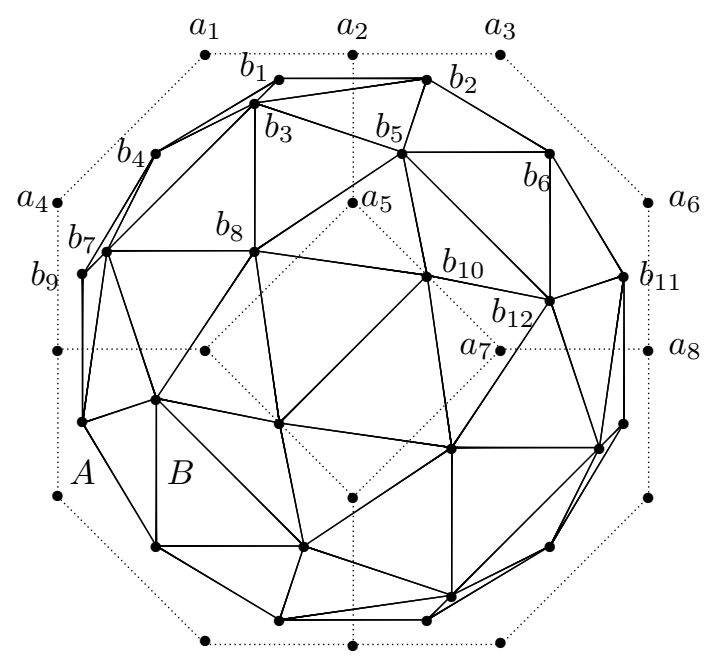

Fig. 1. Truncated octahedron $A$ and the polytope $B$ in Example 4.9

For every edge $k$ of $A$ there exists $f \in\left(\mathbb{R}^{3}\right)^{*}$ such that $H_{f}(B)$ is a translate of $k=$ $H_{f}(A)$. Let $k_{1}=H_{f_{1}}(A), \ldots, k_{36}=H_{f_{36}}(A)$ be all edges of $A$ and $l_{1}=H_{f_{1}}(B), \ldots, l_{36}=$ $H_{f_{36}}(B)$ be respective edges of $B$ and $l_{i}=k_{i}+x_{i}$, where $x_{i} \in \mathbb{R}^{3}, i=1, \ldots, 36$. Let $E$ be a polytope in $\mathbb{R}^{3}$ such that $S_{i}=H_{f_{i}}(E), i=1, \ldots 36$, are all the facets of $E$. Let $y_{1}, \ldots, y_{36}$ be such points in $\mathbb{R}^{3} \backslash(A+E)$ that $(A+E) \cup\left[\left(k_{i}+S_{i}\right) \vee y_{i}\right]$ is convex and $(B+E) \cup\left[\left(l_{i}+S_{i}\right) \vee\left(y_{i}+x_{i}\right)\right]$ is convex for $i=1, \ldots, 36$. Then for $C=(A+E) \vee \bigvee_{i=1}^{36} y_{i}$ and $D=(B+E) \vee \bigvee_{i=1}^{36}\left(y_{i}+x_{i}\right)$ we have $(A, B) \sim(A+E, B+E) \sim(C, D)$. Notice that all edges of $A$ are $k_{i}=H_{f_{i}}(A)$ and all $H_{f_{i}}(C)=y_{i}$ are singletons. Yet $(B, D) \leq(A, C)$ and the pair $(A, C)$ is not minimal. Hence it is not reduced. This example shows that the assumption for all $f \in\left(\mathbb{R}^{n}\right)^{*}$ in Theorem 4.6 is essential.

5. Criteria of minimality. In this section we present two sufficient criteria of minimality for pairs of bounded closed convex sets. The third criterion from [17], the edges criterion, was presented in previous section as Theorem 4.6.

5.1. Facets criterion. First, let us give the following proposition which generalizes Lemma 2 in $[17]$ :

Proposition 5.1. Let $A, B \in \mathcal{B}\left(\mathbb{R}^{n}\right)$, and suppose the set $A$ is a polytope but not a segment, $B \subset A$ and $S_{i}=H_{f_{i}}(A)$, where $i=1, \ldots, k$ are all facets of $A$. If for any $i=1, \ldots, k$ the set $S_{i} \dot{-} H_{f_{i}}(B)$ is empty or $S_{i}$ is a translate of $H_{f_{i}}(B)$ then $A=B$.

Proof. We first assume that bd $A \cap$ bd $B \neq \emptyset$. Since bd $A=\bigcup_{i=1}^{k} S_{i}, a \in S_{i}$ for some $a \in \operatorname{bd} A \cap B$ and $i \in\{1, \ldots, k\}$, we have $a \in H_{f_{i}}(B) \subset H_{f_{i}}(A)=S_{i} \subset H_{f_{i}}(B)-x_{i}$, for some $x_{i} \in \mathbb{R}^{n}$. From the order cancellation law $x_{i}=0$, and, $H_{f_{i}}(B)=S_{i}$. If $S_{i} \cap S_{j} \neq \emptyset$ then there exist $c \in B \cap S_{j}$. Again

$$
c \in H_{f_{j}}(B) \subset H_{f_{j}}(A)=S_{j} \subset H_{f_{j}}(B)-x_{j} .
$$

Therefore, $H_{f_{j}}(B)=S_{j}$ for all $j$ such that $S_{i} \cap S_{j} \neq \emptyset$. Since for any $l \in\{1, \ldots, k\}$ there 
exist $i_{1}, \ldots, i_{m} \in\{1, \ldots, k\}$ such that $S_{i} \cap S_{i_{1}} \neq \emptyset, \ldots, S_{i_{m}} \cap S_{l} \neq \emptyset$, we have $H_{f_{l}}(B)=S_{l}$. We have just proved that bd $A=\bigcup_{i=1}^{k} S_{i} \subset B \subset A$. Since $B$ is convex, $A=B$.

If the boundary bd $A \cap B=\emptyset$ we can expand the set $B$ by homothetic mapping in such a way that the image of $B$ contains $B$, is contained in $A$, and touches the boundary of $A$. Such an image of $B$ satisfies the assumptions of the proposition and, by the former part of the proof, the image of $B$ is equal to $A$.

Thanks to Theorem 15.6 in [4] we can assume in our proposition that $S_{i} \dot{-} H_{f_{i}}(B)=\emptyset$ or $S_{i}$ is a translate of $H_{f_{i}}(B)$ only for all $i=1, \ldots, k-n+1$. Proposition 5.1 helps us to prove the following new theorem which generalizes Theorem 1 in [17].

THEOREM 5.2 (Facets criterion). Let $(X, \tau)$ be a topological vector space and $A \subset X$ be a polytope (not an interval) with facets $S_{i}=H_{f_{i}}(A)(i=1, \ldots, k)$. If a set $B \in \mathcal{B}(X)$ is such that the pairs $\left(S_{i}, H_{f_{i}}(B)\right)$ are minimal for all $i=1, \ldots, k-n+1$, then the pair $(A, B)$ is minimal.

Proof. Let us assume that there exists a pair $\left(A^{\prime}, B^{\prime}\right) \in \mathcal{B}^{2}(X)$ such that $\left(A^{\prime}, B^{\prime}\right) \leq$ $(A, B)$. Thanks to the formula of the addition of faces we deduce from $A+B^{\prime}=B+A^{\prime}$, that for all $i \in\{1, \ldots, k\}$

$$
S_{i}+H_{f_{i}}\left(B^{\prime}\right)=H_{f_{i}}(B)+H_{f_{i}}\left(A^{\prime}\right) .
$$

By Theorem 2.1 there exists a minimal pair $(C, D)$ such that

$$
(C, D) \leq\left(H_{f_{i}}\left(A^{\prime}\right), H_{f_{i}}\left(B^{\prime}\right)\right) \sim\left(S_{i}, H_{f_{i}}(B)\right) .
$$

Since both pairs $(C, D)$ and $\left(S_{i}, H_{f_{i}}(B)\right)$ are equivalent and minimal for each $i \in\{1, \ldots$, $k-n+1\}$, the set $S_{i}-C$ is empty or $S_{i}$ is a translate of $C$. Since $C \subset H_{f_{i}}\left(A^{\prime}\right)$, the set $S_{i} \dot{-} H_{f_{i}}\left(A^{\prime}\right)$ is empty or $H_{f_{i}}\left(A^{\prime}\right)$ is a translate of $S_{i}$. Now, by generalized Proposition 5.1 , we have $A^{\prime}=A$. Hence, by the cancellation law, $B^{\prime}=B$.

The following theorem from [15] follows as a corollary to Theorem 5.2.

TheOrem 5.3. Let $A, B \in \mathcal{B}\left(\mathbb{R}^{n}\right)$ and $A$ be a polytope with nonempty interior. Let $H_{f}(B)$ be a singleton for each facet $H_{f}(A)$. Then the pair $(A, B)$ is minimal.

Corollary 5.4. Let $A, B \in \mathcal{B}\left(\mathbb{R}^{3}\right)$ and $A$ be a polytope with nonempty interior and facets $S_{i}=H_{f_{i}}(A)(i=1, \ldots, k)$. If $B$ is such the set that the pair $\left(S_{i}, H_{f_{i}}(B)\right)$ is minimal $i=1, \ldots, k$, then the pair $(A, B)$ is minimal.

The corollary follows from Theorem 5.2 and from the fact that a minimal pair in a two-dimensional space always has the property of translation [10], [32].

Theorem 5.2 generalizes of the following result of Ch. Bauer ([3], Theorem 5.1):

Theorem 5.5. Let $A, B \in \mathcal{B}\left(\mathbb{R}^{n}\right)$ and $A, B$ be polytopes with $\operatorname{dim}(A+B)=n$. Let $H_{f_{i}}(A+B), i=1, \ldots, k$ be all facets of $A+B$. If the pair $\left(H_{f_{i}}(A), H_{f_{i}}(B)\right)$ is minimal for all $i=1, \ldots, k-n+1$ then the pair $(A, B)$ is minimal.

We still do not possess necessary and sufficient conditions for minimality of a pair of three-dimensional polytopes. 
5.2. Steps criterion. For $A, B \in \mathcal{B}(X)$, let $A-B=A+(-B)$. First, we present the following lemma:

Lemma 5.6. Let $X$ be a Hausdorff topological vector space, $A, B, A^{\prime}, B^{\prime} \in \mathcal{B}(X), a \in$ $A, b \in B$ and let $A^{\prime}$ and $B^{\prime}$ be compact. Let $\left(A^{\prime}, B^{\prime}\right) \leq(A, B)$ and $a+b \in \exp (A+$ $B), a-b \in \operatorname{ext}(\mathrm{A}-\mathrm{B})$. Then $a+b \in \exp \left(A^{\prime}+B^{\prime}\right), a \in A^{\prime}, b \in B^{\prime}$.

Proof. The sets $A+B, A-B$ do not have to be closed. Since $A^{\prime}, B^{\prime}$ are compact, $A+B^{\prime}=$ $B+A^{\prime}$. For some $f \in X^{*},\{a+b\}=H_{f}(A+B)$. Then $H_{f}(A)+H_{f}\left(B^{\prime}\right)=H_{f}(B)+$ $H_{f}\left(A^{\prime}\right), H_{f}(A)=\{a\}, H_{f}(B)=\{b\}$. Therefore,

$$
a+H_{f}\left(B^{\prime}\right)=b+H_{f}\left(A^{\prime}\right) .
$$

Given any $b^{\prime} \in H_{f}\left(B^{\prime}\right)$, then $a+b^{\prime}=b+a^{\prime}$ for some $a^{\prime} \in H_{f}\left(A^{\prime}\right)$. But $a-b=a^{\prime}-b^{\prime} \in$ $\operatorname{ext}(A-B)$ implies $a=a^{\prime}$ and $b=b^{\prime}$. Hence $a+b \in A^{\prime}+B^{\prime} \subset A+B, a+b \in \exp (A+B)$. Since $A^{\prime}+B^{\prime} \subset A+B, a+b \in \exp \left(A^{\prime}+B^{\prime}\right)$.

Theorem 5.7 (Steps criterion, [17]). Let $X$ be a normed space and let $(A, B) \in \mathcal{K}^{2}(X)$. If for every $a_{0}+b_{0} \in \exp (A+B)$ there exists a sequence $\left(a_{i}+b_{i}\right)_{i=1}^{k} \subset \exp (A+B)$ such that $a_{k}-b_{k} \in \operatorname{ext}(A-B)$ and for every $i, a_{i-1}=a_{i}$ or $b_{i-1}=b_{i}$, then the pair $(A, B)$ is minimal.

Proof. Let $\left(A^{\prime}, B^{\prime}\right) \leq(A, B)$ and $a_{0}+b_{0} \in \exp (A+B)$. Then there exists a sequence $\left(a_{i}+b_{i}\right)_{i=1}^{k} \subset \exp (A+B)$ with $a_{k}-b_{k} \in \operatorname{ext}(A-B)$. From Lemma 5.6 we obtain $a_{k}+b_{k} \in$ $\exp \left(A^{\prime}+B^{\prime}\right), a_{k} \in A^{\prime}, b_{k} \in B^{\prime}$. Suppose that $a_{k-1}=a_{k}$. Hence $a_{k-1}+b_{k-1} \in A^{\prime}+B=$ $B^{\prime}+A \subset A+B$. Since $a_{k-1}+b_{k-1} \in \exp (A+B), a_{k-1}+b_{k-1} \in \exp \left(A+B^{\prime}\right)$. Thus $b_{k-1} \in$ $B^{\prime}$ and $a_{k-1}+b_{k-1} \in \exp \left(A^{\prime}+B^{\prime}\right)$. If $b_{k-1}=b_{k}$ then $a_{k-1}+b_{k-1} \in \exp \left(A^{\prime}+B^{\prime}\right)$, too. Similarly, we can show step by step that $a_{i}+b_{i} \in \exp \left(A^{\prime}+B^{\prime}\right)$ for $i=k-2, k-3, \ldots 1,0$. Now, using a theorem of Klee, [23], we have $A+B=\overline{\operatorname{conv}} \exp (A+B) \subset A^{\prime}+B^{\prime} \subset A^{\prime}+B$. Hence $A^{\prime}=A$ and $B^{\prime}=B$.

The following proposition illustrates special mutual positions of sets $A, B$ such that $a+b \in \exp (A+B), a-b \in \operatorname{ext}(A-B)$ for some $a \in A, b \in B$.

Proposition 5.8. Let $(A, B) \in \mathcal{B}^{2}\left(\mathbb{R}^{n}\right), n \geq 2, a+b \in \exp (A+B)$, and $a-b \in \operatorname{ext}(A-$ $B)$. Then there exist halfspaces $H_{f}^{-}=\left\{x \in \mathbb{R}^{n} \mid f(x) \leq 0\right\}, H_{g}^{-}=\left\{x \in \mathbb{R}^{n} \mid g(x) \leq 0\right\}$, and $H_{g}^{+}=\left\{x \in \mathbb{R}^{n} \mid g(x) \geq 0\right\}$ associated with functionals $f, g \in\left(\mathbb{R}^{n}\right)^{*}$ such that $A \subset\left(a+H_{f}^{-}\right) \cap\left(a+H_{g}^{-}\right)$and $B \subset\left(b+H_{f}^{-}\right) \cap\left(b+H_{g}^{+}\right)$. Moreover, $A \backslash\{a\} \subset a+\operatorname{int} H_{f}^{-}$ and $B \backslash\{b\} \subset b+\operatorname{int} H_{f}^{-}$.

Proof. Let $\{a+b\}=H_{f}(A+B)$ for some $f \in\left(\mathbb{R}^{n}\right)^{*}$. Then $\{a\}=H_{f}(A),\{b\}=H_{f}(B)$. For any $c \in A \backslash\{a\}$ we have $f(c-a)<0$. Hence $A \backslash\{a\} \subset a+\operatorname{int} H_{f}^{-}$. Similarly we can show that $B \backslash\{b\} \subset b+\operatorname{int} H_{f}^{-}$.

On the other hand $a-b \in H_{g}(A-B)$ for some $g \in\left(\mathbb{R}^{n}\right)^{*}$. Then $a \in H_{g}(A),-b \in$ $H_{g}(-B)=-H_{-g}(B)$. For any $c \in A$ we have $g(c-a) \leq 0$, and for any $c \in B$ we have $-g(c-b) \leq 0$. Hence $A \subset a+H_{g}^{-}$and $B \subset a+H_{g}^{+}$.

Figure 2 illustrates the last proposition. 


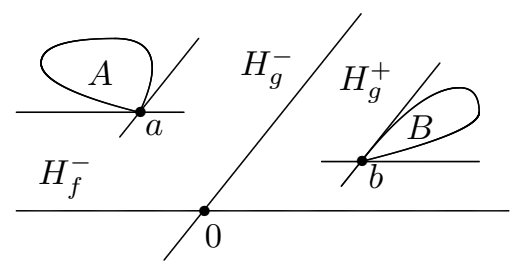

Fig. 2. The sets $A$ and $B$ in Proposition 5.8

EXAmple 5.9. Let $A$ and $B$ be the convex polygons in Figure 3.

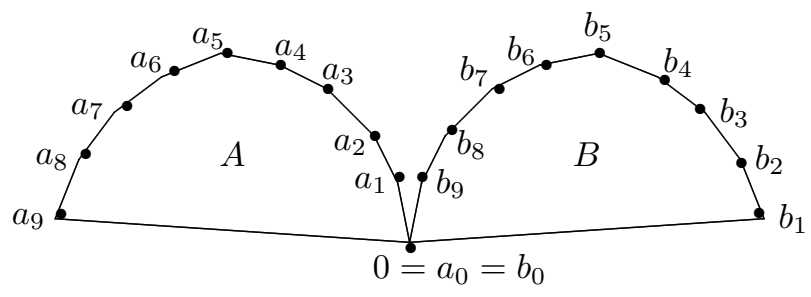

Fig. 3. Polygons $A$ and $B$ in Example 5.9

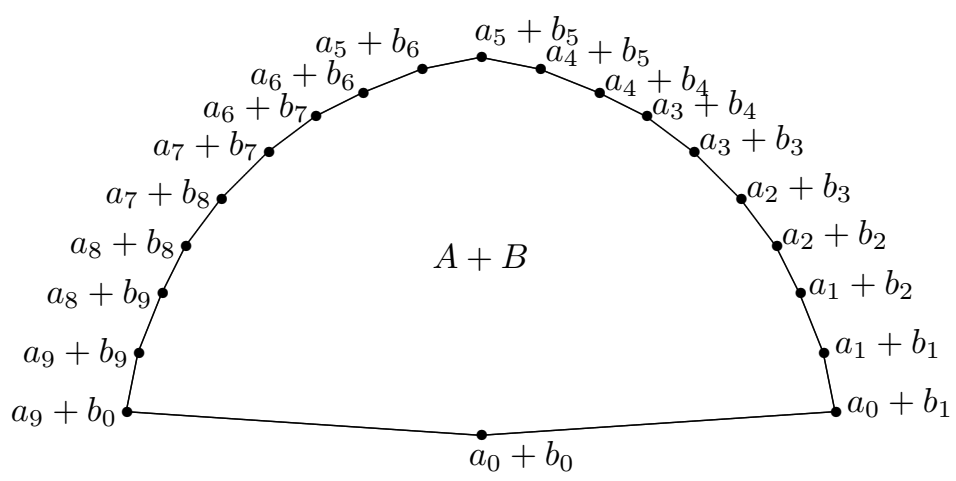

Fig. 4. Polygon $A+B$ in Example 5.9

Let us observe that the point $a_{0}+b_{0}$ is the only vertex of $A+B$ such that $a_{0}-b_{0}$ is a vertex of $A-B$. By Theorem 5.7 the pair $(A, B)$ is minimal.

ExAmple 5.10. In Figure 5 the polytopes $A$ and $B$ are two pyramids with parallel triangular bases and with vertices $a_{4}$ and $b_{4}$ such that $A$ is much taller than $B$.

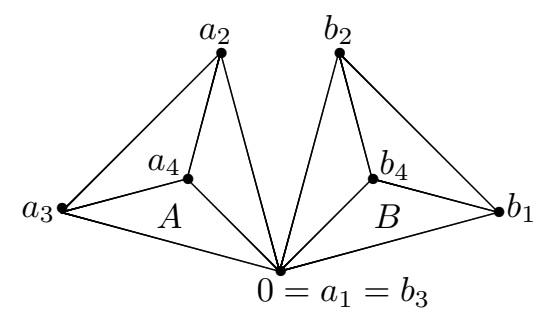

Fig. 5. Pyramids $A$ and $B$ in Example 5.10 


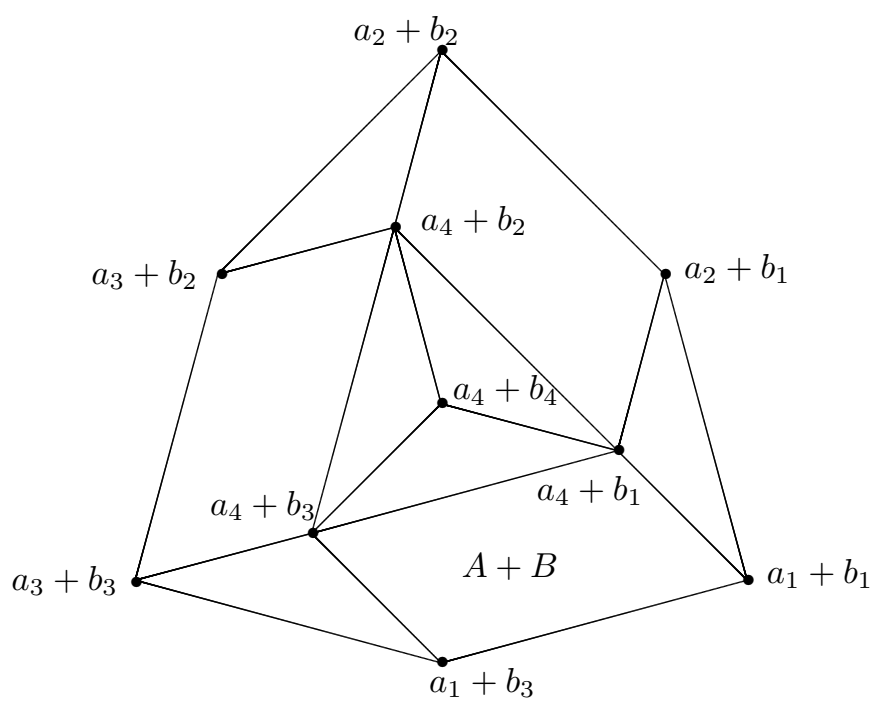

Fig. 6. Sum of pyramids $A$ and $B$ in Example 5.10

The sum $A+B$ has a hexagonal base and the vertices $a_{4}+b_{2}, a_{4}+b_{3}$ and $a_{4}+b_{1}$ belong to the plane parallel to the base of $A+B$. By Theorem 5.7 the pair $(A, B)$ is minimal.

6. Reduction methods. Theorem 3.3 provides us with a general method of finding minimal pairs in any quotient class $[A, B] \in \widetilde{\mathbb{R}^{2}}$.

In the case of convex polygons, M. Handschug, [19], presented an algorithm for finding a minimal pair equivalent to any given pair of convex polygons. M. Wiernowolski modified the algorithm of Handschug and prepared a computer program in [36]. Unfortunately, we have neither a general method of finding minimal pairs for all quotient classes $[A, B] \in$ $\widetilde{\mathbb{R}^{3}}$ nor an algorithm of finding a minimal pair equivalent to any given pair of threedimensional polytopes.

In this section we present a partial solution to the problem of finding a smaller, if not minimal, pair equivalent to the given one. The most natural method of finding a smaller equivalent pair is presented in the following simple proposition:

Proposition 6.1 (Reduction by summand). If the sets $A, B \in \mathcal{B}(X)$ have a common summand $C \in \mathcal{B}(X)$ then $(A \dot{-} C, B \dot{-} C) \in[A, B]$.

The following propositions present a method of reducing pairs of sets by removing identical parts of them:

Proposition 6.2. Let $A, B, F, P \in \mathcal{B}(X), B=A \cup P$ and $F=A \cap P$. Then the pairs $(A, B)$ and $(F, P)$ are equivalent. 


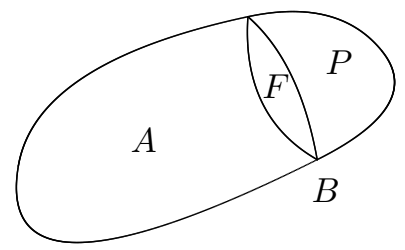

Fig. 7

Proof. By Theorem 1.1 we obtain $A \dot{+} P=A \cup P \dot{+} A \cap P$.

Corollary 6.3. Let $B \in \mathcal{B}(X)$ and $H$ be a closed hyperplane dividing the space $X$ into two closed halfspaces $H^{+}$and $H^{-}$such that $A \cap H$ is nonempty. Let $A=B \cap H^{-}, P=$ $B \cap H^{+}$and $F=B \cap H$. Then the pairs $(A, B)$ and $(F, P)$ are equivalent.

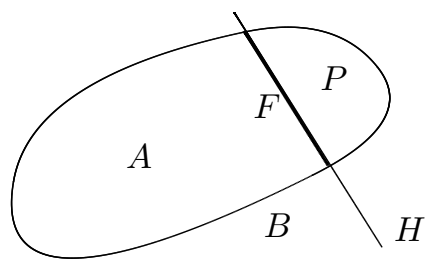

Fig. 8

By Corollary 6.3 we conclude that parts of two bounded closed convex sets which can be translated onto each other can be cut off by hyperplanes without leaving the quotient class.

Proposition 6.4. Let $A, B, F, G, P, Q \in \mathcal{B}(X), B=A \cup P \cup Q$, the sets $A \cup P$ and $A \cup Q$ be convex, $F=A \cap P, G=A \cap Q$ and $P \cap Q \subset A$. Then the pairs $(A, B)$ and $(F+G, P \dot{+} Q)$ are equivalent.

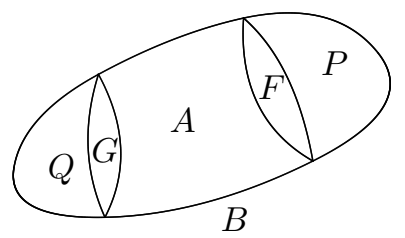

Fig. 9

Proof. Since the sets $A \cup P$ and $A \cup Q$ are convex, we have $A+P=A \cup P \dot{+} F$ and $A \dot{+} Q=A \cup Q \dot{+} G$. Adding these equations we obtain $A \dot{+} A \dot{+} P \dot{+} Q=A \cup P \dot{+} A \cup Q \dot{+} F \dot{+}$ $G=B \dot{+}(A \cup P) \cap(A \cup Q) \dot{+} F \dot{+} G=B \dot{+} A \cup(P \cap Q) \dot{+} F \dot{+} G=B \dot{+} A \dot{+} F \dot{+} G$. Applying the cancellation law, we get $A \dot{+} P \dot{+} Q=B \dot{+} F \dot{+} G$. Hence $(A, B) \sim(F \dot{+} G, P \dot{+} Q)$.

Corollary 6.5. Let $B \in \mathcal{B}(X)$ and $H_{1}, H_{2}$ be closed hyperplanes such that $B \cap H_{1}^{+} \cap$ $H_{2}^{-} \subset H_{1} \cap H_{2}$. Let $A=B \cap H_{1}^{-} \cap H_{2}^{+}, P=B \cap H_{1}^{+}, Q=B \cap H_{2}^{-}, F=B \cap H_{1}$ and $G=B \cap H_{2}$. Then the pairs $(A, B)$ and $(F \dot{+} G, P \dot{+} Q)$ are equivalent. 


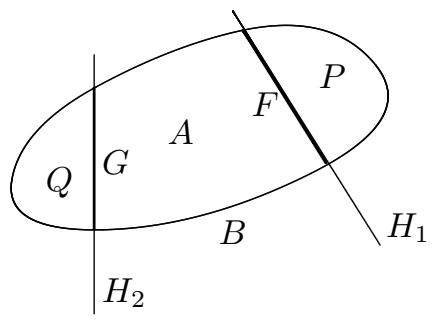

Fig. 10

Proof. Let us notice that $A \cup P=\left(B \cap H_{1}^{-} \cap H_{2}^{+}\right) \cup\left(B \cap H_{1}^{+}\right)=\left(B \cap H_{1}^{-} \cap H_{2}^{+}\right) \cup(B \cap$ $\left.H_{1}^{+} \cap H_{2}^{+}\right) \cup\left(B \cap H_{1}^{+} \cap H_{2}^{-}\right)$. Since $B \cap H_{1}^{+} \cap H_{2}^{-} \subset B \cap H_{1} \cap H_{2} \subset B \cap H_{1}^{+} \cap H_{2}^{+}$, we have $A \cup P=\left(B \cap H_{2}^{+} \cap H_{1}^{-}\right) \cup\left(B \cap H_{2}^{+} \cap H_{1}^{+}\right)=B \cap H_{2}^{+}$. Hence the set $A \cup P$ is convex. Similarly, $A \cup Q$ is convex, too. Moreover, the condition $B \cap H_{1}^{+} \cap H_{2}^{-} \subset H_{1} \cap H_{2}$ is equivalent to $B \cap H_{1}^{+} \cap H_{2}^{-} \subset A$. Then we apply the proposition.

Let us notice that in general the pair $(F \dot{+} G, P \dot{+} Q)$ does not have to be smaller than $(A, B)$.

EXAMPLE 6.6. Let us consider the following pair $(A, B)$ :

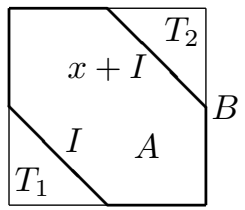

Fig. 11

The set $A$ is a hexagon, $B$ is a square, $T_{1}$ and $T_{2}$ are triangles and $I$ is a segment. Applying Proposition 6.4, we obtain an equivalent pair $\left(I+I+x, T_{1}+T_{2}\right)$ :

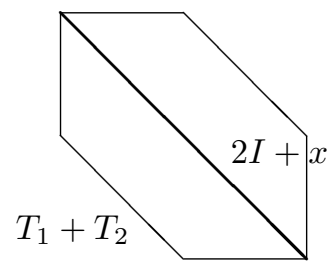

Fig. 12

Since $\left(T_{1}+x\right) \cup T_{2}$ is convex, $T_{1}+x+T_{2}=\left(T_{1}+x\right) \cup T_{2}+I+x$. Hence $\left(I+I+x, T_{1}+T_{2}\right) \sim$ $\left(I+x,\left(T_{1}+x\right) \cup T_{2}\right):$

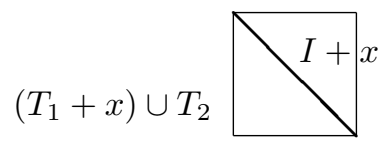

Fig. 13

Let us consider the following sets $A, B, P, Q, F, G$ : 


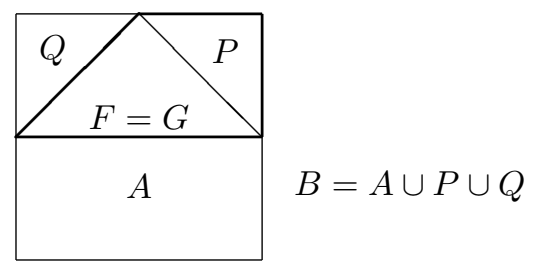

Fig. 14

The set $A$ is a rectangle, $P$ and $Q$ are trapezoids, $F=G=A \cap P=A \cap Q$ is a segment and $P \cap Q$ is a triangle. These sets fulfill all assumptions of Proposition 6.4 but $P \cap Q \nsubseteq A$. The reader can prove that the pairs $(A, B)$ and $(F+G, P+Q)$ are not equivalent. In fact, here the pair $(A, B)$ is equivalent to a pair consisting of a singleton and a segment.

\section{Number of equivalent minimal pairs}

EXAMPLE 7.1. In [10] we presented the first example of two equivalent minimal pairs of compact convex subsets in $\mathbb{R}^{3}$ which are not related by translation. The example is as follows:
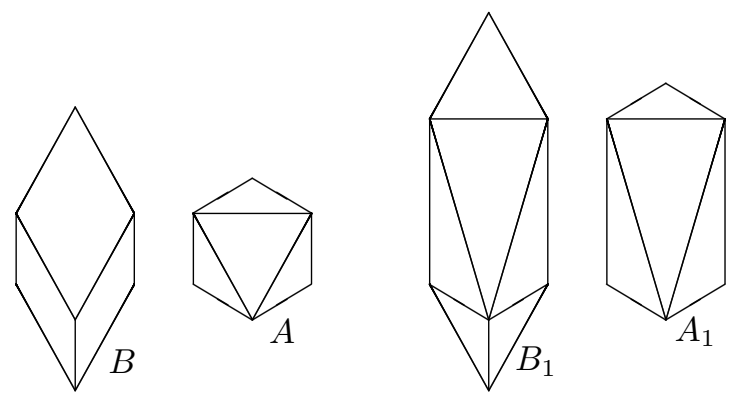

Fig. 15

Let $a, b, c, d \in \mathbb{R}^{3}$ be given by

$$
a=\left(\frac{1}{\sqrt{3}}, 0,0\right), \quad b=\left(-\frac{1}{2 \sqrt{3}}, \frac{1}{2}, 0\right), \quad c=\left(-\frac{1}{2 \sqrt{3}},-\frac{1}{2}, 0\right), \quad d=\left(0,0, \frac{\sqrt{2}}{\sqrt{3}}\right) .
$$

Let us define the set $T=a \vee b \vee c$ and the sets

$$
A=T \vee(d-T), \quad B=-d \vee A \vee 2 d, \quad A_{1}=T \vee(2 d-T), \quad B_{1}=-d \vee A_{1} \vee 3 d
$$

Let us denote $F=d-T, P=(d-T) \vee 2 d, G=T$ and $Q=T \vee(-d)$. By Proposition 6.4 the pairs $(A, B)$ and $(F+G, P+Q)=(T-T+d,(-T) \vee(T-T+d) \vee(T+2 d))$ are equivalent. Notice that the last pair is the pair $(C, D)$ from Figure 16. Applying Proposition 6.4 to the pair $\left(A_{1}, B_{1}\right)$ we obtain that the latter pair is equivalent to the pair $(T-T+2 d,(-T+d) \vee(T-T+2 d) \vee(T+3 d))=(C+d, D+d)$. Therefore, the pairs $(A, B)$ and $\left(A_{1}, B_{1}\right)$ are equivalent. 

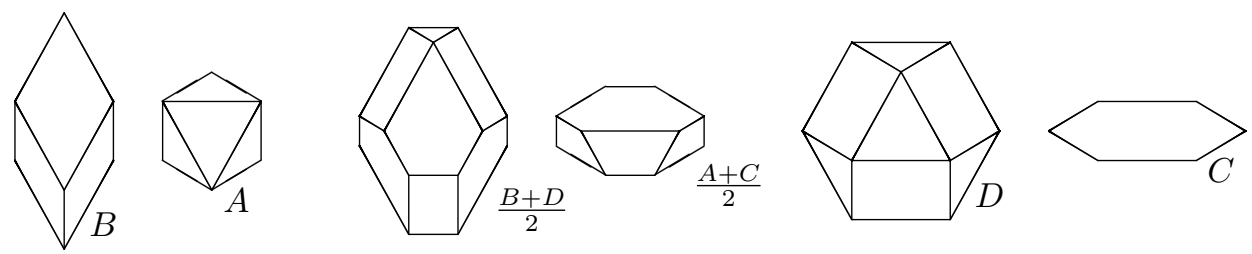

Fig. 16

Both pairs $(A, B)$ and $\left(A_{1}, B_{1}\right)$ are minimal by Proposition $7.2(\mathrm{i})$; the pair $(C, D)$ is minimal by Proposition 7.2(ii).

Proposition 7.2. Let $X$ be a Hausdorff topological vector space and $f \in X^{*} \backslash\{0\}$. Let $F, F_{1}, G, G_{1} \in \mathcal{B}(X), F, F_{1}, G, G_{1}$ be compact subsets of $f^{-1}(0), A=F \vee\left(G+x_{2}\right)$, $B=A \vee\left(F_{1}+x_{1}\right) \vee\left(G_{1}+x_{3}\right), C=F+G$ and $D=C \vee\left(F+G_{1}+x_{3}-x_{2}\right) \vee\left(G+F_{1}+x_{1}\right)$, where $x_{1}, x_{2}, x_{3} \in X, f\left(x_{1}\right)<0<f\left(x_{2}\right)<f\left(x_{3}\right)$ and

$$
\begin{aligned}
& f\left(x_{2}\right)\left(F_{1}+x_{1}\right)-f\left(x_{1}\right)\left(G+x_{2}\right) \subset f\left(x_{2}-x_{1}\right) F, \\
& f\left(x_{3}-x_{2}\right) F+f\left(x_{2}\right)\left(G_{1}+x_{3}\right) \subset f\left(x_{3}\right)\left(G+x_{2}\right) .
\end{aligned}
$$

(i) If the pairs $\left(F, F_{1}\right),\left(G, G_{1}\right)$ are minimal and have the property of translation and $F \dot{-} G=G \dot{-} F=\emptyset$, then the pair $(A, B)$ is minimal.

(ii) If $f\left(x_{3}\right)-f\left(x_{2}\right) \leq f\left(x_{2}\right),-f\left(x_{1}\right) \leq f\left(x_{2}\right)$ and the pair $\left(F+G_{1}, G+F_{1}\right)$ is minimal and has the property of translation then the pair $(C, D)$ is minimal.

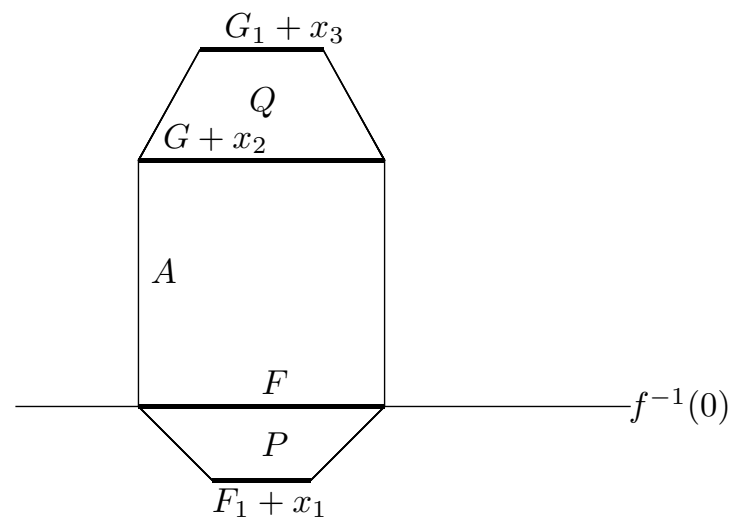

Fig. 17

Proof. (i) Suppose that $\left(A^{\prime}, B^{\prime}\right) \leq(A, B)$ for some $\left(A^{\prime}, B^{\prime}\right) \in \mathcal{B}^{2}(X)$. Hence $A+B^{\prime}=$ $B+A^{\prime}$. We have

$$
H_{f}(A)=G+x_{2}, H_{f}(B)=G_{1}+x_{3}, H_{-f}(A)=F, H_{-f}(B)=F_{1}+x_{1} .
$$

Since $H_{f}(A)+H_{f}\left(B^{\prime}\right)=H_{f}(B)+H_{f}\left(A^{\prime}\right)$ and $H_{-f}(A)+H_{-f}\left(B^{\prime}\right)=H_{-f}(B)+H_{-f}\left(A^{\prime}\right)$, we have

$G+x_{2}+H_{f}\left(B^{\prime}\right)=G_{1}+x_{3}+H_{f}\left(A^{\prime}\right)$ and $F+H_{-f}\left(B^{\prime}\right)=F_{1}+x_{1}+H_{-f}\left(A^{\prime}\right)$.

Hence $\left(G+x_{2}, G_{1}+x_{3}\right) \sim\left(H_{f}\left(A^{\prime}\right), H_{f}\left(B^{\prime}\right)\right)$ and $\left(F, F_{1}+x_{1}\right) \sim\left(H_{-f}\left(A^{\prime}\right), H_{-f}\left(B^{\prime}\right)\right)$. Since the pair $\left(G, G_{1}\right)$ is minimal and has the property of translation, $G+x_{2}+z \subset H_{f}\left(A^{\prime}\right)$ and $G_{1}+x_{3}+z \subset H_{f}\left(B^{\prime}\right)$ for some $z \in X$. Therefore, $G+z+x_{2} \subset A$. 
Since $F \dot{-} G=\emptyset$ and $A=F \vee\left(G+x_{2}\right), G+z+x_{2} \subset G+x_{2}$ and $z=0$. Hence $G+x_{2} \subset A^{\prime}$.

In a similar way, taking $-f$ instead of $f$, we prove that $F \subset A^{\prime}$. Then $A=F \vee(G+$ $\left.x_{2}\right) \subset A^{\prime} \subset A$. Hence $A^{\prime}=A$ and by the cancellation law $B^{\prime}=B$.

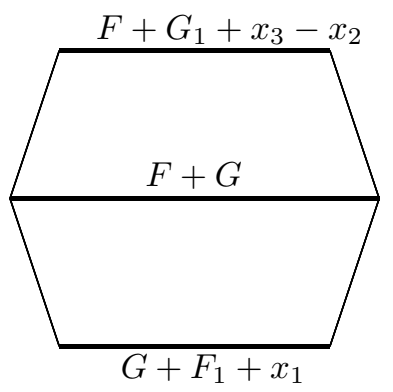

Fig. 18

(ii) Suppose that $\left(A^{\prime}, B^{\prime}\right) \leq(C, D)$. We have $A^{\prime}+D=B^{\prime}+C$ and $H_{f}\left(A^{\prime}\right)+H_{f}(D)=$ $H_{f}\left(B^{\prime}\right)+H_{f}(C), H_{-f}\left(A^{\prime}\right)+H_{-f}(D)=H_{-f}\left(B^{\prime}\right)+H_{-f}(C)$. Hence $A^{\prime}+F+G_{1}+x_{3}-x_{2}=$ $H_{f}\left(B^{\prime}\right)+F+G$ and $A^{\prime}+G+F_{1}+x_{1}=H_{-f}\left(B^{\prime}\right)+F+G$. By the cancellation law we have $A^{\prime}+G_{1}+x_{3}-x_{2}=H_{f}\left(B^{\prime}\right)+G$ and $A^{\prime}+F_{1}+x_{1}=H_{-f}\left(B^{\prime}\right)+F$.

After adding these equalities we obtain $A^{\prime}+G_{1}+x_{3}-x_{2}+H_{-f}\left(B^{\prime}\right)+F=A^{\prime}+F_{1}+$ $x_{1}+H_{f}\left(B^{\prime}\right)+G$.

Again by the cancellation law we obtain $G_{1}+F+H_{-f}\left(B^{\prime}\right)+x_{3}-x_{2}=G+F_{1}+$ $H_{f}\left(B^{\prime}\right)+x_{1}$. Hence $\left(F+G_{1}, G+F_{1}\right) \sim\left(H_{f}\left(B^{\prime}\right)+x_{1}, H_{-f}\left(B^{\prime}\right)+x_{3}-x_{2}\right)$.

Since the pair $\left(F+G_{1}, G+F_{1}\right)$ is minimal and has the property of translation, $F+G_{1}+x \subset H_{f}\left(B^{\prime}\right)+x_{1}$ and $G+F_{1}+x \subset H_{-f}\left(B^{\prime}\right)+x_{3}-x_{2}$ for some $x \in X$.

By $A^{\prime}+G_{1}+x_{3}-x_{2}=H_{f}\left(B^{\prime}\right)+G$ and the order law of cancellation we obtain $A^{\prime} \supset F+G+x-x_{1}+x_{2}-x_{3}=C+x-x_{1}+x_{2}-x_{3}$.

Since $A^{\prime} \subset C, x-x_{1}+x_{2}-x_{3}=0$ and $A^{\prime}=C^{\prime}$. Obviously, $B^{\prime}=D$.

Let us mention that in $\mathbb{R}^{3}$ there exists a continuum of equivalent minimal pairs which are not related by translations. The complete example of such a family of equivalent minimal pairs can be found in [26]. The construction is based on the notion of a general frustum, which was introduced by G. T. Sallee [30]. By definition, a general frustum is the convex hull of two convex sets that lie in different parallel hyperplanes. We will restrict ourselves to the case of compact general frusta which can be defined as follows:

Let $X$ be a real locally convex topological vector space, $f \in X^{*}$ a continuous linear functional, $z \in X$, with $f(z) \neq 0$ and $E, F \in \mathcal{K}(X)$ a nonempty compact convex set with $E, F \subset f^{-1}(0)$. Then

$$
A=E \vee(F+z)
$$

is called a general frustum over $E$ and $F$.

Fixing the continuous linear functional, $f \in X^{*}$ and the point $z \in X$, with $f(z) \neq 0$, we use the notation

$$
A=E \vee(F+z)
$$

for a general frustum over $E$ and $F$. 
In the following theorem (Theorem 4.3 in [26]) we construct explicitly a family of continuum many equivalent minimal pairs $\left(A_{z}, B_{z}\right) \in \mathcal{B}^{2}\left(\mathbb{R}^{3}\right), z=\left(z_{1}, z_{2}, z_{3}\right) \in\left(\mathbb{R}_{+}\right)^{3}$ which are not related by translations, i.e. for $z, z^{\prime} \in\left(\mathbb{R}_{+}\right)^{3}, z \neq z^{\prime}$ there exists no element $x \in \mathbb{R}^{3}$ such that

$$
A_{z}+x=A_{z^{\prime}} \text { and } B_{z}+x=B_{z^{\prime}} .
$$

THEOREM 7.3. For $z \in\left(\mathbb{R}_{+}\right)^{3}$ define the following sets:

i) $T_{0}=(1,0,0) \vee(0,1,0) \vee(0,0,1), T_{1}=(1,1,0) \vee(1,0,1) \vee(0,1,1)$,

ii) $S_{0}=T_{0} \vee(0,0,0), S_{1}=T_{1} \vee(1,1,1)$,

iii) $A_{z}=T_{0} \vee\left(T_{1}+z\right), B_{z}=S_{0} \vee\left(S_{1}+z\right)$.

Then the family $\left\{\left(A_{z}, B_{z}\right) \mid z \in\left(\mathbb{R}_{+}\right)^{3}\right\}$ is a family of equivalent minimal pairs which are not related by translations.

Theorem 7.3 is a simple consequence of Proposition 6.4 and 7.2(i).
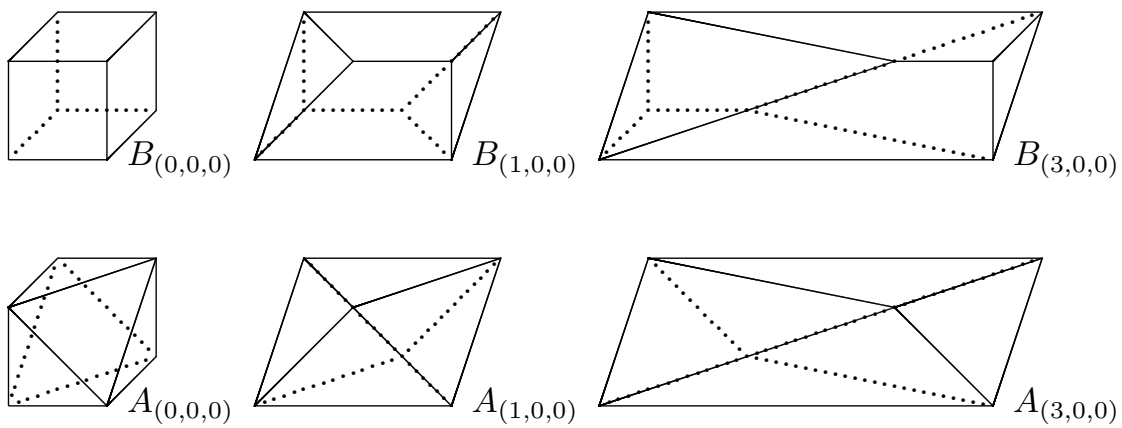

Fig. 19

Let us notice that for $z=\left(z_{1}, 0,0\right)$ both sets $A_{z}, B_{z}$ are frusta (see Figure 19). Notice that we get minimal pairs of arbitrarily large diameter. Until now we have not been able to describe all the family of minimal pairs in $\left[A_{(0,0,0)}, B_{(0,0,0)}\right]$ (or $[A, B]$ from Example 7.1).

Let $(A, B) \in \mathcal{B}^{2}(X)$ and $m[A, B]$ be the family of all elements of $[A, B]$ that are minimal with respect to the ordering " $\leq$ ", and $n_{A, B}$ be the number of minimal pairs in $m[A, B]$ that are not translates of one another. If $X$ is a one or two-dimensional space then $n_{A, B}$ is always equal to 1 ([10], [32]). In Theorem 7.3 there are given $A, B \in \mathcal{B}\left(\mathbb{R}^{3}\right)$ such that $n_{A, B}$ is equal to the continuum.

In December 2000, Professor S. Rolewicz posed the problem of whether $n_{A, B}$ can be finite and greater than 1 . The following Theorem (see [14]) gives the negative answer to the problem.

THEOREM 7.4. Let $\left(A_{1}, B_{1}\right),\left(A_{2}, B_{2}\right)$ be two equivalent minimal pairs of compact convex sets such that $\left(A_{2}, B_{2}\right)$ is not a translate of $\left(A_{1}, B_{1}\right)$. Then there exists an uncountable family $\left(A_{\lambda}, B_{\lambda}\right), \lambda \in \Lambda$ of minimal pairs that are equivalent to $\left(A_{1}, B_{1}\right)$ and no $\left(A_{\lambda}, B_{\lambda}\right)$ is a translate of $\left(A_{\mu}, B_{\mu}\right), \lambda \neq \mu$.

Let us notice that for the two minimal pairs $(A, B)$ and $\left(A_{1}, B_{1}\right)$ from Example 7.1 and a number $t, 0<t<1$ the equivalent pair $\left((1-t) A+t A_{1},(1-t) B+t B_{1}\right)$ is never 
minimal. On the other hand the equivalent pair $((1-t) A+t C,(1-t) B+t D)$ is always minimal.

\section{Application of minimal pairs}

8.1. Space of classes of asymmetry. Let $A, B \in \mathcal{B}(X)$. We define a new equivalence relation in $\mathcal{B}(X)$ by

$$
A \approx B \Leftrightarrow A \dot{+}(-B)=B \dot{+}(-A) .
$$

By $[A]$ we denote the quotient class of $A$, the so called asymmetry class. The set of asymmetry classes with the addition $[A]+[B]=[A \dot{+} B]$ and the multiplication $\alpha \cdot[A]=$ $\left[\alpha^{+} A+\alpha^{-}(-A)\right]$ is a vector space.

G. Ewald and G. Shephard ([8]) proved that in the space of the asymmetry classes over $\mathbb{R}^{2}$, for every class $[A]$, there exists a set $B \in \mathcal{B}\left(\mathbb{R}^{2}\right)$ such that $[A]=\{B+C \mid C \in$ $\mathcal{B}\left(\mathbb{R}^{2}\right)$ and $\left.C=-C\right\}$. In other words, every minimal (with respect to the inclusion) element of asymmetry class is reduced.

M. Wiernowolski in [37] showed that in the space of asymmetry classes over the topological vector space $X$ the set $A$ is a minimal element of its asymmetry class $[A]$, if and only if the pair $(A,-A)$ is minimal.

In fact, the pair $(A,-A) \in \mathcal{B}^{2}\left(\mathbb{R}^{2}\right)$ is minimal, if and only if it is reduced.

8.2. Kernel of an element of the Minkowski-Rådström-Hörmander space. The following theorem given in [9] is a consequence of the uniqueness up to translation of minimal pairs in $\mathbb{R}^{2}$

TheOREM 8.1. Let $\left(A_{0}, B_{0}\right) \in \mathcal{B}^{2}\left(\mathbb{R}^{2}\right)$,

$$
C=\bigcap_{(A, B) \in\left[A_{0}, B_{0}\right]}(A+(-B)) \text { and } D=\bigcap_{(A, B) \in\left[A_{0}, B_{0}\right]}(B+(-B)) \text {. }
$$

Then the kernel $(C, D)$ of the quotient class $\left[A_{0}, B_{0}\right]$ belongs to $\left[A_{0}, B_{0}\right]$. If the pair $\left(A_{0}, B_{0}\right)$ is minimal then $C=A_{0}+\left(-B_{0}\right), D=B_{0}+\left(-B_{0}\right)$.

The following example from [16] shows that in $\mathbb{R}^{3}$ the kernel of the quotient class $\left[A_{0}, B_{0}\right]$ may or may not belong to the quotient class $\left[A_{0}, B_{0}\right]$.

ExAmPLE 8.2. Let $T=(1,0,0) \vee(0,1,0) \vee(0,0,1)$ and $a=(1,1,1) . A_{0}=(-T) \vee(T-$ $T) \vee T$ and $B_{0}=T-T$.
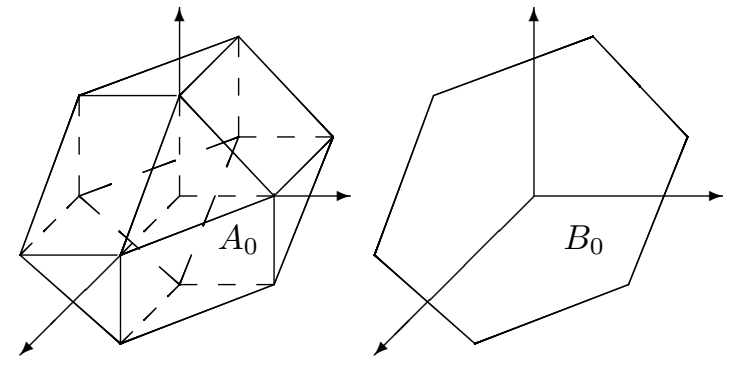

Fig. 20 
In this example the pair $\left(A_{0}, B_{0}\right)$ is equal to the kernel of the class $\left[A_{0}, B_{0}\right]$. Since $A_{0}=$ $-A_{0}, B_{0}=-B_{0}$, the kernel of the class $\left[B_{0}, A_{0}\right]$ is equal to $\left(A_{0}, C\right)$ for some $C \in \mathcal{B}\left(\mathbb{R}^{3}\right)$. On the other hand, since $B_{0}$ is not a summand of $2 A_{0}, 2 A_{0} \neq B_{0}+C$. Thus $\left(A_{0}, C\right)$ does not belong to the class $\left[B_{0}, A_{0}\right]$.

8.3. Representation of continuous selections of linear functions in $\mathbb{R}^{3}$. Let $U \subset \mathbb{R}^{n}$ be an open subset and $f, f_{1}, \ldots, f_{m}: U \rightarrow \mathbb{R}$ be continuous functions. If $I(x)=\{i \in$ $\left.\{1, \ldots, m\} \mid f_{i}(x)=f(x)\right\}$ is nonempty at every point $x \in U$, then $f$ is called a continuous selection of the functions $f_{1}, \ldots, f_{m}$. We denote by $C S\left(f_{1}, \ldots, f_{m}\right)$ the set of all continuous selections of $f_{1}, \ldots, f_{m}$. The set $I(x)$ is called the active index set of $f$ at the point $x$. The functions $f_{1}, \ldots, f_{m}$ will be called generating functions.

Typical examples of continuous selections are the functions

$$
f_{\max }=\max \left(f_{1}, \ldots, f_{m}\right), f_{\min }=\min \left(f_{1}, \ldots, f_{m}\right)
$$

or, more generally, any finite superposition of maximum and minimum operations over subsets of the functions $f_{1}, \ldots, f_{m}$.

In [22] the notion of a nondegenerate critical point for a continuous selection of $C^{2}$ functions has been defined and it has been shown that a continuous selection $f$ of $C^{2}$ functions is topologically equivalent to a function of the form

$$
y \rightarrow f\left(x_{0}\right)+g\left(y_{1}, \ldots, y_{k}\right)-\sum_{i=k+1}^{k+\mu} y_{i}^{2}+\sum_{j=k+\mu+1}^{n} y_{j}^{2}
$$

in a neighbourhood of a nondegenerate critical point $x_{0}$, where $k=\left|I\left(x_{0}\right)\right|-1, g \in$ $C S\left(y_{1}, \ldots, y_{k},-\sum_{i=1}^{k} y_{i}\right)$, and $\mu$ is the quadratic index of $f$ at $x_{0}$. For more details see [21], Chapter 7, and [22].

In [2] it has been shown that every continuous selection of linear functions $l_{1}, \ldots, l_{m}$ on $\mathbb{R}^{n}$ has a representation of the form

$$
l(x)=\min _{i \in\{1, \ldots, r\}} \max _{j \in M_{i}} l_{j}(x),
$$

where $M_{i} \subset\{1, \ldots, m\}$ and that this representation is unique, provided the linear functions are affinely independent, i.e. $\sum_{i=1}^{m} \lambda_{i} l_{i}=0, \sum_{i=1}^{m} \lambda_{i}=0$ implies that $\lambda_{i}=0$, and $M_{i} \subset M_{j}$ if and only if $i=j$. Note that in particular the functions $l_{i}(x)=x_{i}, i=$ $1, \ldots, n, l_{n+1}(x)=-\sum_{i=1}^{n} x_{i}$ are affinely independent. The topological structure of a continuous selection of $C^{2}$ functions in the vicinity of a nondegenerate critical point is thus completely determined by its quadratic index $\mu$ and a unique collection of index sets $M_{1}, \ldots, M_{r}$. This fact has been used in [1] to extend the classical smooth Morse theory to piecewise smooth functions.

In Theorem 8.3 (see [12]) we find minimal pairs of compact convex sets (polytopes) that represent 166 (see [2]) continuous selections in $C S\left(l_{1}, l_{2}, l_{3}, l_{4}\right)$ in $\mathbb{R}^{3}$.

THEOREM 8.3. Let $a, b, c, d \in \mathbb{R}^{3}$ be affinely independent vectors such that $a+b+c+d=0$. Then $C S(\langle a, \cdot\rangle,\langle b, \cdot\rangle,\langle c, \cdot\rangle,\langle d, \cdot\rangle)$ consists of differences of support functions $p_{A}-p_{B}$ where the minimal pair $(A, B)$ is one of the 16 types presented in the table below. 
Table. Types of minimal pairs

$\mathbb{O}=(a+b) \vee(a+c) \vee(b+c) \vee(a+d) \vee(b+d) \vee(c+d),-a \vee b=-(a \vee b)$

\begin{tabular}{|c|c|c|c|}
\hline Type & $\begin{array}{l}\text { The pair } \\
\text { consists of }\end{array}$ & $\begin{array}{c}\text { Number } \\
\text { of pairs } \\
\text { in the type }\end{array}$ & $\begin{array}{l}\text { Examples } \\
\text { of minimal pairs }\end{array}$ \\
\hline 1 & two singletons & 4 & $(\{a\},\{0\}),(\{b\},\{0\}),(\{c\},\{0\}),(\{d\},\{0\})$ \\
\hline 2 & $\begin{array}{c}\text { segment } \\
\text { and singleton }\end{array}$ & 12 & $(a \vee b,\{0\}),(\{0\},-a \vee b)$ \\
\hline 3 & $\begin{array}{c}\text { triangle } \\
\text { and singleton }\end{array}$ & 8 & $\begin{array}{l}(a \vee b \vee c,\{0\}) \\
(\{0\},-a \vee b \vee c)\end{array}$ \\
\hline 4 & $\begin{array}{l}\text { triangle } \\
\text { and segment }\end{array}$ & 24 & $\begin{array}{c}(-a \vee b \vee c,-a \vee b-c) \\
(a \vee b+c, a \vee b \vee c)\end{array}$ \\
\hline 5 & two triangles & 4 & $(-a \vee b \vee c-d, a \vee b \vee c)$ \\
\hline 6 & $\begin{array}{l}\text { tetrahedron } \\
\text { and singleton }\end{array}$ & 2 & $\begin{array}{l}(a \vee b \vee c \vee d,\{0\}) \\
(\{0\},-a \vee b \vee c \vee d)\end{array}$ \\
\hline 7 & $\begin{array}{c}\text { pyramid } \\
\text { and segment }\end{array}$ & 12 & $\begin{array}{c}((a \vee b+c \vee d) \vee(a+b), a \vee b), \\
(-a \vee b,(a \vee b+c \vee d) \vee(c+d))\end{array}$ \\
\hline 8 & $\begin{array}{l}\text { octahedron } \\
\text { and triangle }\end{array}$ & 8 & $\begin{array}{l}(\mathbb{O}, a \vee b \vee c) \\
(-a \vee b \vee c, \mathbb{O})\end{array}$ \\
\hline 9 & $\begin{array}{c}\text { octahedron } \\
\text { and tetrahedron }\end{array}$ & 2 & $\begin{array}{l}(\mathbb{O}, a \vee b \vee c \vee d) \\
(-a \vee b \vee c \vee d, \mathbb{O})\end{array}$ \\
\hline 10 & $\begin{array}{l}\text { pyramid } \\
\text { and triangle }\end{array}$ & 24 & $\begin{array}{l}((b \vee c+d \vee a) \vee(a+d)), a \vee b \vee c), \\
(-a \vee b \vee c,(b \vee c+d \vee a) \vee(b+c))\end{array}$ \\
\hline 11 & $\begin{array}{l}\text { pyramid } \\
\text { and tetrahedron }\end{array}$ & 12 & $\begin{array}{l}((a \vee b+c \vee d) \vee(c+d), a \vee b \vee c \vee d), \\
(-a \vee b \vee c \vee d,(a \vee b+c \vee d) \vee(c+d))\end{array}$ \\
\hline 12 & $\begin{array}{l}\text { tetrahedron } \\
\text { and triangle }\end{array}$ & 8 & $\begin{array}{c}(-a \vee b \vee c \vee d,-a \vee b \vee c-d) \\
(a \vee b \vee c+d, a \vee b \vee c \vee d)\end{array}$ \\
\hline 13 & $\begin{array}{l}\text { tetrahedron } \\
\text { and square }\end{array}$ & 6 & $\begin{array}{c}(-a \vee b \vee c \vee d, a \vee c+b \vee d) \\
(a \vee c+b \vee d, a \vee b \vee c \vee d)\end{array}$ \\
\hline 14 & $\begin{array}{l}\text { truncated } \\
\text { rhombohedron } \\
\text { and prism }\end{array}$ & 24 & $\begin{array}{c}(\mathbb{O} \vee(-2 a), a \vee b \vee c-a \vee d+d) \\
(a \vee d-a \vee b \vee c-d, \mathbb{O} \vee 2 a) \\
\text { (see Figure 21) }\end{array}$ \\
\hline 15 & two prisms & 12 & $\begin{array}{c}(d \vee c-a \vee b \vee c-d, a \vee c+b \vee c \vee d) \\
\text { (see Figure 23) }\end{array}$ \\
\hline 16 & $\begin{array}{l}\text { two truncated } \\
\text { rhombohedra }\end{array}$ & 4 & $\begin{array}{c}((\mathbb{O}+d) \vee(-d), \mathbb{O} \vee 2 d) \\
(\text { see Figure } 25)\end{array}$ \\
\hline
\end{tabular}

Three out of 16 types are minimal pairs that are not unique-up-to-translation minimal representations in their own quotient classes.

Assuming that $a, b, c, d$ are the vertices of a regular tetrahedron with the center at the origin, a pair of Type 14 is presented in Figure 21. 

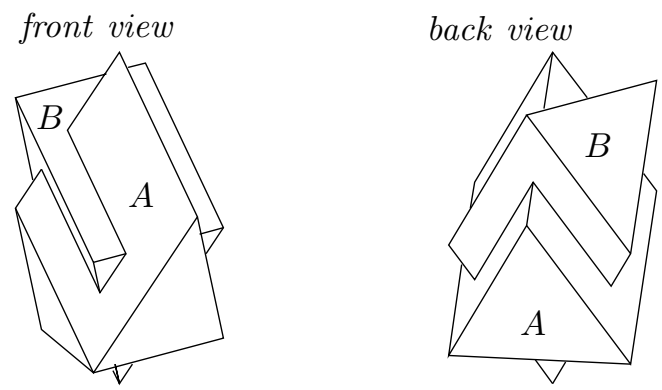

Fig. 21

The minimal pair $(C, D)$ in Figure 22 is equivalent to $(A, B)$ from Figure 21.
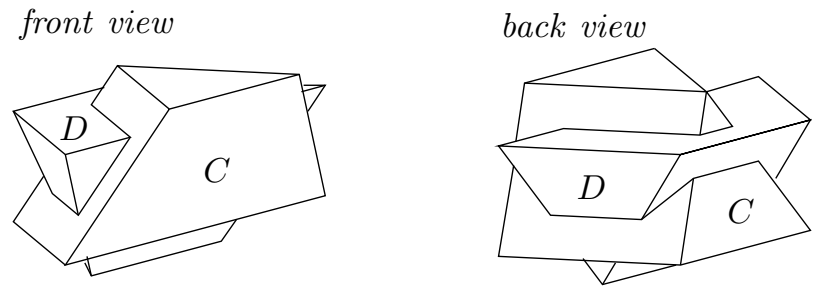

Fig. 22

A minimal pair of Type 15 is presented in Figure 23.
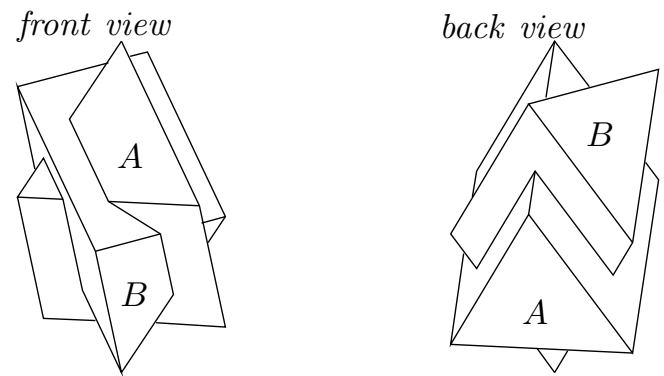

Fig. 23

The minimal pair $(C, D)$ in Figure 24 is equivalent to $(A, B)$ from Figure 23 . There exists an axis such that for rotation $R$ by 180 degrees around it we have $C=R(A), D=$ $R(B)$.

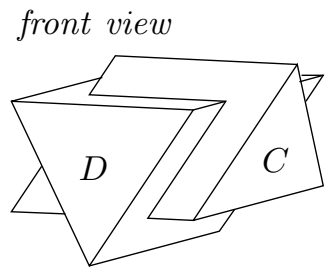

back view

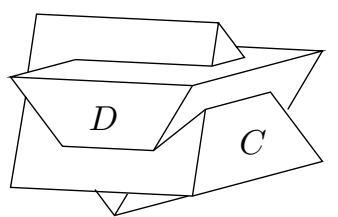

Fig. 24 
Equivalent minimal pairs $(A, B)$ of Type 16 in Figure 25 and $(C, D)$ in Figure 26 are related to the pairs given in Example 7.1 and depicted in Figure 16.
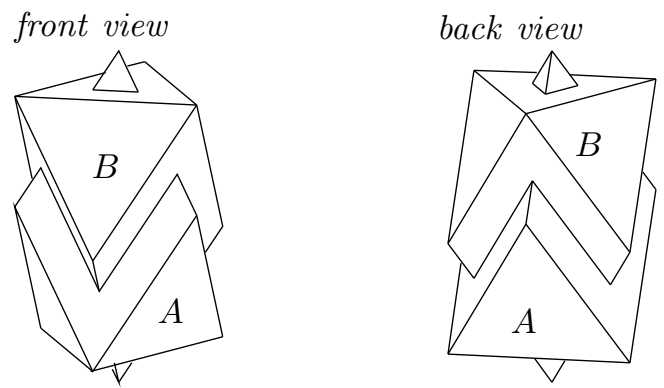

Fig. 25
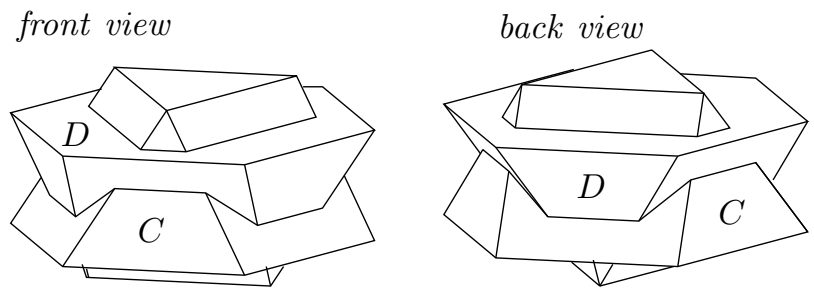

Fig. 26

The pairs of Types 1-10,12 are minimal and have the property of translation. We do not know whether pairs of Types 11,13 have the property of translation or not. Pairs of Types 1-3,5-6 are reduced. All other pairs are not reduced (see Theorem 4.3).

\section{References}

[1] A. A. Agrachev, D. Pallaschke and S. Scholtes, On Morse theory for piecewise smooth functions, Journal of Dynamical and Control Systems 3 (1997), 449-469.

[2] S. G. Bartels, L. Kuntz and S. Scholtes, Continuous selections of linear functions and nonsmooth critical point theory, Nonlinear Analysis, Theory, Meth. \& Appl. 24 (1995), 385-407.

[3] Ch. Bauer, Minimal and reduced pairs of convex bodies, Geom. Dedicata 62 (1996), 179192.

[4] A. Brøndsted, An Introduction to Convex Polytopes, Springer-Verlag, Heidelberg,1983.

[5] V. F. Demyanov and A. M. Rubinov, Quasidifferential Calculus, Optimization Software Inc., Publications Division, New York 1986.

[6] V. F. Demyanov and A. M. Rubinov, Foundations of Nonsmooth Analysis and Quasidifferential Calculus, Optimization and Operations Research 23, Nauka, Moscow 1990.

[7] V. F. Demyanov and A. M. Rubinov, Constructive Nonsmooth Analysis, Verlag Peter Lang, Frankfurt/M 1995.

[8] G. Ewald and G. Shephard, Normed vector spaces consisting of classes of convex sets, Math. Zeitschr. 91 (1966), 1-19. 
[9] Y. Gao, Z. Q. Xia and L. W. Zhang, Kernelled quasidifferential for a quasidifferentiable function in two-dimensional space, J. Convex Anal. 8 (2001), 401-408.

[10] J. Grzybowski, Minimal pairs of convex compact sets, Arch. Math. 63 (1994), 173-181.

[11] J. Grzybowski, A note on summands of compact convex sets, Mathematika 43 (1996), 286-294.

[12] J. Grzybowski, D. Pallaschke and R. Urbański, Minimal pairs representing selections of four linear functions in $\mathbb{R}^{3}$, J. Convex Anal. 7 (2000), 445-452.

[13] J. Grzybowski and R. Urbański, Minimal pairs of bounded closed convex sets, Studia Math. 126 (1997), 95-99.

[14] J. Grzybowski and R. Urbański, On the number of minimal pairs of compact convex sets that are not translates of one another, Studia Math. 158 (2003), 59-63.

[15] J. Grzybowski and R. Urbański, On reduced pairs of bounded closed convex sets, Rev. Mat. Complut. 16 (2003), 555-560.

[16] J. Grzybowski and R. Urbański, On the representation property of kernels of quasidifferentials, J. Convex Anal. 11 (2004), 387-390.

[17] J. Grzybowski and R. Urbański, Three criteria of minimality for pairs of compact convex sets, Optimization 55 (2006), 569-576.

[18] J. Grzybowski, R. Urbański and M. Wiernowolski, On common summands and antisummands of compact convex sets, Bull. Polish Acad. Sci. Math. 47 (1999), 69-76.

[19] M. Handschug, On equivalent quasidifferentials in the twodimensional case, Optimization 20 (1989), 37-43.

[20] L. Hörmander, Sur la fonction d'appui des ensembles convexes dans un espace localement convexe, Arkiv för Matematik 3 (1954), 181-186.

[21] H. Th. Jongen, P. Jonker and F. Twilt, Nonlinear Optimization in $\mathbb{R}^{n}$, I. Morse Theory, Chebyshev Approximation, Peter Lang Verlag, Frankfurt, 1983.

[22] H. Th. Jongen and D. Pallaschke, On Linearization and continuous selections of functions, Optimization 19 (1998), 343-353.

[23] V. Klee, Extremal structure of convex sets, II, Math. Zeitschr. 69 (1958), 90-104.

[24] D. Pallaschke, S. Scholtes and R. Urbański, On minimal pairs of compact convex sets, Bull. Polish Acad. Sci. Math. 39 (1991), 1-5.

[25] D. Pallaschke and R. Urbański, Reduction of quasidifferentials and minimal representations, Math. Programming (Series A) 66 (1994), 161-180.

[26] D. Pallaschke and R. Urbański, A continuum of minimal pairs of compact convex sets which are not connected by translations, J. Convex Analysis 3 (1996), 83-95.

[27] D. Pallaschke and R. Urbański, Pairs of Compact Convex Sets, Fractional Arithmetic with Convex Sets, Mathematics and Its Applications, Kluwer, Dordrecht, 2002.

[28] A. G. Pinsker, The space of convex sets of a locally convex space, Trudy Leningrad Engineering-Economic Institute 63 (1966), 13-17.

[29] H. Rådström, An embedding theorem for spaces of convex sets, Proc. Amer. Math. Soc. 3 (1952), 165-169.

[30] G. T. Sallee, On the indecomposibility of the cone, J. London Math. Soc. 9 (1974), 363-367.

[31] S. Schneider, Convex Bodies: The Brunn-Minkowski Theory, Encyclopedia of Mathematics and its Applications 44, Cambridge University Press, 1993.

[32] S. Scholtes, Minimal pairs of convex bodies in two dimensions, Mathematika 39 (1992), $267-273$.

[33] S. Scholtes, On convex bodies and some applications to optimization, Doctoral Thesis, University of Karlsruhe, 1992. 
[34] R. Urbański, A generalization of the Minkowski-Rådström-Hörmander Theorem, Bull. Acad. Polon. Sci. Sér. Sci. Math. Astr. Phys. 24 (1976), 709-715.

[35] R. Urbański, On minimal convex pairs of convex compact sets, Archiv der Mathematik 67 (1996), 226-238.

[36] M. Wiernowolski, Examination of the minimality of pairs of compact convex sets, Master Thesis, Adam Mickiewicz University, Poznań, 1992.

[37] M. Wiernowolski, Minimality in asymmetry classes, Studia Math. 124 (1997), 149-154. 
